\title{
Measuring the unknown: an estimator and simulation study for assessing case reporting during epidemics
}

\section{Short title: Estimating reporting of cases in disease outbreaks}

\author{
Christopher I Jarvis ${ }^{1,2}$, Amy Gimma ${ }^{1,2}$, Flavio Finger ${ }^{1,2,3}$, Tim P Morris ${ }^{4}$, Jennifer A Thompson ${ }^{1}$, \\ Olivier le Polain de Waroux ${ }^{2,5}$, W John Edmunds ${ }^{1,2}$, Sebastian Funk ${ }^{1,2}$, \\ Thibaut Jombart ${ }^{1,2,6,7}$ \\ ${ }^{1}$ Department of Infectious Disease Epidemiology, London School of Hygiene \& Tropical Medicine, \\ London, United Kingdom \\ ${ }^{2}$ Centre for Mathematical Modelling of Infectious Diseases, London School of Hygiene \& Tropical \\ Medicine, London, UK. \\ ${ }^{3}$ Epicentre, Paris, France \\ ${ }^{4}$ MRC Clinical Trials Unit at UCL, London, United Kingdom \\ ${ }^{5}$ Public Health England, London, United Kingdom \\ ${ }^{6}$ MRC Centre for Global Infectious Disease Analysis, Department of Infectious Disease Epidemiology, \\ School of Public Health, Imperial College London, United Kingdom \\ ${ }^{7}$ UK Public Health Rapid Support Team, London, United Kingdom
}

\section{Corresponding authors:}

Christopher I Jarvis

Department of Infectious Disease Epidemiology London School of Hygiene \& Tropical Medicine

Keppel Street

London WC1E 7HT

United Kingdom

christopher.jarvis@Ishtm.ac.uk

Thibaut Jombart

Department of Infectious Disease Epidemiology London School of Hygiene \& Tropical Medicine

Keppel Street

London WC1E 7HT

United Kingdom

thibautjombart@gmail.com 


\section{Abstract}

The fraction of cases reported, known as 'reporting', is a key performance indicator in an outbreak response, and an essential factor to consider when modelling epidemics and assessing their impact on populations. Unfortunately, its estimation is inherently difficult, as it relates to the part of an epidemic which is, by definition, not observed.

We introduce a simple statistical method for estimating reporting, initially developed for the response to Ebola in Eastern Democratic Republic of the Congo (DRC), 2018-2020. This approach uses transmission chain data typically gathered through case investigation and contact tracing, and uses the proportion of investigated cases with a known, reported infector as a proxy for reporting. Using simulated epidemics, we study how this method performs for different outbreak sizes and reporting levels. Results suggest that our method has low bias, reasonable precision, and despite sub-optimal coverage, usually provides estimates within close range $(5-10 \%)$ of the true value.

Being fast and simple, this method could be useful for estimating reporting in real-time in settings where person-to-person transmission is the main driver of the epidemic, and where case investigation is routinely performed as part of surveillance and contact tracing activities. 


\section{Author summary}

When responding to epidemics of infectious diseases, it is essential to estimate how many cases are not being reported. Unfortunately reporting, the proportion of cases actually observed, is difficult to estimate during an outbreak, as it typically requires large surveys to be conducted on the affected populations. Here, we introduce a method for estimating reporting from case investigation data, using the proportion of cases with a known, reported infector. We used simulations to test the performance of our approach by mimicking features of a recent Ebola epidemic in the Democratic Republic of the Congo. We found that despite some uncertainty in smaller outbreaks, our approach can be used to obtain informative ballpark estimates of reporting under most settings. This method is simple and computationally inexpensive, and can be used to inform the response to any epidemic in which transmission events can be uncovered by case investigation. 


\section{Introduction}

The response to infectious disease outbreaks increasingly relies on the analysis of various data sources to inform operation in real time [1,2]. Outbreak analytics can be used to characterise key factors driving epidemics, such as transmissibility, severity, or important delays like the incubation period or the serial interval [2]. Amongst these factors, the amount of infections remaining undetected in the affected populations is a crucial indicator for assessing the state of an epidemic, and yet this quantity is often hard to estimate in real time [3-6]. Indeed, estimation of the overall proportion of individuals infected (attack rates) typically requires time-consuming serological surveys [7-9] which may not be achievable in resource-limited, large-scale emergencies such as the 2014-2016 Ebola virus disease (EVD) outbreak in West Africa [10], or the more recent EVD outbreak in Eastern provinces of the Democratic Republic of the Congo (DRC) $[11,12]$.

As an alternative, one may attempt to quantify reporting, i.e. the proportion of all infections which result in notified cases. Unfortunately, this quantity is also hard to estimate, and usually requires the analysis of epidemiological and genomic data through complex methods for reconstructing transmission trees [13-15] or transmission clusters [16]. Such requirements can mean that by the time estimates are available, decisions have already been made, or the outbreak situation has changed [17-19]. Therefore, approaches for timely estimation of reporting to help inform decision making during a response are required.

Methods for estimating reporting in real time should ideally exploit data which is routinely collected as part of the outbreak response. In diseases where dynamics are mostly governed by 
person-to-person transmission, case investigation and contact tracing can be powerful tools for understanding past transmission events as well as detecting new cases as early as possible $[11,20-23]$. For vaccine-preventable diseases, contact tracing can also be used for designing ring vaccination strategies, as seen in recent EVD outbreaks in the DRC $[11,20]$. These data also contain information about reporting. Intuitively, the frequency of cases whose infector is a known and reported case is indicative of the level of reporting: the more frequently case investigation identifies a known infector, the higher the corresponding case reporting should be. Conversely, cases with no known epidemiological link after investigation are indicative of unobserved infections, and therefore under-reporting.

In this article, we introduce a method to estimate case reporting from contact tracing data. This approach, designed during the Ebola outbreak in Eastern DRC [11,12], was originally aimed at assessing case reporting in a context where insecurity made surveillance difficult, and under-reporting likely [12]. The approach utilized transmission chain data and calculated the proportion of cases with a known epidemiological link as a proxy for reporting. We provide a derivation of the estimator and explain the rationale of this approach and assess its performance using simulated outbreaks of different sizes with varying levels of reporting. Based on the simulation results, we make some suggestions regarding the use of this method to inform strategic decision making during an outbreak response. 


\section{Methods}

We present the analytical derivation of our method of estimating reporting, defined as the proportion of cases actually notified during an outbreak. We then describe the simulation study, using the ADEMP (Aim, Data generating mechanism, Estimand, Methods, Performance measures) framework as described by Morris et al 2019 [24,25], used to evaluate the performance of the methods under various conditions.

\section{Estimating reporting from epidemiological links}

Our method exploits transmission chains derived from case investigation and contact tracing data. The rationale for the approach is to consider the proportion of cases with a known infector as a proxy for the proportion of infections (including asymptomatic but infectious individuals) reported. The proportion of cases with a known infector is by definition the proportion of infectors who were reported (Figure 1), so that the reporting probability $\pi$ can be estimated as $\widehat{\pi}=\frac{n_{k}}{n_{k}+n_{u}}$ where $n_{k}$ is the number of secondary cases (infectees) with a known infector and $n_{u}$ is the number of secondary cases without a known infector.

\section{Derivation of estimator for reporting}

We define

$m_{r}$ - number of reported infectors

$m_{u}$ - number of unreported infectors

$n_{k}$ - number of secondary cases (infectees) with known infector

$n_{u}$ - number of secondary cases without known infector 
$R$ - reproduction number, i.e. average number of secondary cases by case

$\pi$ - reporting probability following some unspecified probability distribution with unknown

probability parameter such that $E(\pi)=\frac{m_{r}}{m_{r}+m_{u}}$ where secondary cases are assumed to follow the same reporting distribution as primary infections.

Let $n_{u}+n_{k}$ be the total number of secondary cases following an unspecified probability distribution so that $E\left(n_{u}+n_{k}\right)=\left(m_{r}+m_{u}\right) R$.

Then the expected number of reported infectees with a known infector is

$$
E\left(n_{k}\right)=m_{r} R \pi
$$

Similarly, the expected number of reported infectees without a known infector is

$$
E\left(n_{u}\right)=m_{u} R \pi
$$

From this we have that

$$
m_{r}=\frac{E\left(n_{k}\right)}{R \pi} \text { and } m_{u}=\frac{E\left(n_{u}\right)}{R \pi}
$$

By definition

$$
\pi=\frac{m_{r}}{m_{r}+m_{u}}
$$

Therefore

$$
E(\pi)=\frac{\frac{E\left(n_{k}\right)}{R \pi}}{\frac{E\left(n_{k}\right)+E\left(n_{u}\right)}{R \pi}}=\frac{E\left(n_{k}\right)}{E\left(n_{k}\right)+E\left(n_{u}\right)}
$$

and replacing the expectations with their estimates from the data, we get the estimator

$$
\widehat{\pi}=\frac{n_{k}}{n_{k}+n_{u}} .
$$




\section{Uncertainty for reporting}

The uncertainty associated with this estimation can be estimated using various methods for computing confidence intervals of proportions. Using the standard approach for estimating standard errors for a proportion we have

$$
S E_{\pi}=\frac{\widehat{\pi} \times(1-\widehat{\pi})}{n_{k}+n_{u}}
$$

Here, we used exact binomial confidence intervals which can be calculated:

$$
\left(1+\frac{n-n_{k}+1}{n_{k} F\left[\frac{\alpha}{2} ; 2 n_{k}, 2\left(n-n_{k}+1\right)\right]}\right)^{-1}<\pi<\left(1+\frac{n-n_{k}}{n_{k} F\left[1-\frac{g}{2} ; 2\left(n_{k}+1\right), 2\left(n-n_{k}\right)\right]}\right)^{-1}
$$

Where $n=n_{k}+n_{u}$, total number of secondary cases. $F\left(c ; d_{1}, d_{2}\right)$ is the $c$ quantile from an F-distribution with $d_{1}, d_{2}$ degrees of freedom and $1-\alpha$ is the confidence level.

\section{Simulation study}

\section{Aim}

We aim to test the performance of the method for different outbreak sizes and actual reporting, in terms of bias, coverage, and precision (in an operational context) using simulated outbreaks.

\section{Data generating mechanism}

We considered twelve data-generating mechanisms (three reporting rates by four reported outbreaks sizes) and performed 4000 repetitions per mechanism.

Each repetition corresponded to a hypothetical outbreak with a known transmission tree. To simulate the reporting process, cases were removed randomly from the transmission chains 
using a Binomial process with a probability (1 - reporting). We will thus distinguish the total outbreak size, which represents all cases in the outbreak, and the reported outbreak size, which represents the number of cases reported. For simplicity, we assumed that all cases reported were investigated, so that it is known if they had a documented epidemiological link, or not, amongst reported cases.

For each outbreak (repetition) we removed observations so that reporting was $25 \%, 50 \%$, or $75 \%$. Therefore a single simulated outbreak will give three different observed outbreaks. We categorised the simulations into reported outbreak sizes of 1-99, 1-499, 500-999, 1000+.

\section{Outbreak simulation}

We used the R package simulacr [26] to simulate outbreaks, the reporting process, and the subsequently observed transmission chains. simulacr implements and extends individual-based simulations of epidemics previously used to evaluate transmission tree reconstruction methods $[13,14,27]$. A Poisson branching process is used to generate new cases in time (with daily time-steps), drawing from a distribution for the reproduction number $(R)$ and the infectious period to determine rates of infection. The infectiousness of a given individual $i$ at time $t$ is, noted $\lambda_{i, t}$, is calculated as:

$$
\lambda_{i, t}=R_{i} \mathrm{w}\left(t-s_{i}\right)
$$

where $R_{i}$ is the reproduction number for individual $i, s_{i}$ is their date of symptom onset, and $w$ is the probability mass function of the duration of infectiousness (time interval between onset of symptom and new secondary infections). New cases generated at time $t+1$ are drawn from a Poisson distribution with a rate $\Lambda_{t}$ summing the infectiousness of all cases:

$$
\Lambda_{t}=\left(n_{s} / n\right) \sum_{i} \Lambda_{i, t}
$$


where $n_{s}$ is the number of susceptible individuals and $n$ the total population size, so that the branching process includes a density-dependence in which rates of infection decrease with the proportion of susceptibles.

Transmission trees are built by assigning infectors to newly infected individuals according to a multinomial distribution in which potential infectors have a probability $\lambda_{i, t} / \sum_{i} \lambda_{i, t}$ of being drawn. The dates of symptom onset and case notification are generated for each new case using user-provided distributions for the incubation time and reporting delays. Simulations run until any of the set duration of the simulation is reached (here, 365 days).

Here, we used parameters values and distributions in line with estimates from the Eastern DRC Ebola outbreak [12,28], the details of which are provided in Table 1. All code used for running these simulations is available from https://github.com/jarvisc1/2020-reporting.

\section{Estimand: Reporting}

We considered a single estimand $\pi$ the level of reporting.

\section{Method}

For each repetition we calculated the proportion of the number of cases with a known infector over the total number of reported cases, that is the estimator $\widehat{\pi}=\frac{n_{k}}{n_{k}+n_{u}}$. We further calculated the standard error and 95\% exact binomial confidence intervals. 


\section{Performance measures}

The performance of the method was measured using bias, coverage, and precision. For bias and coverage, the Monte-Carlo standard errors were calculated to quantify uncertainty about the estimates of the performance [29]. The equations used are detailed in Table 2 and were taken from Morris et al [24]. In addition, results were classified according to different ranges of absolute error, for a more operational interpretation of the results.

Bias is the difference between the expected value and the true value. It was measured by taking the difference between the average estimate of reporting versus the true reporting.

Unbiasedness is a desirable statistical quality but a small amount of bias may be tolerated in exchange for other desirable qualities of an estimator. The estimates of reporting were presented visually by displaying the estimates of all 4000 simulations for each scenario.

Coverage is the percentage of Cls containing the true value. In the case of a $95 \% \mathrm{Cl}$ this should contain the true value $95 \%$ of the time. We counted the number of repetitions where the true value was contained in the $95 \% \mathrm{Cl}$ and divided by the total number of repetitions. The coverage was visualised through the use of Zip plots. This new visualisation created by Morris et al [24], helps to assess the coverage of a method by viewing the Cls directly. Assessing an expected $95 \%$ coverage with a Monte-Carlo standard error of 0.35 requires 3877 simulations [24] which is well within our 4000 simulations . 
Precision represents how close the estimates are to each other. The model based and empirical standard error were also calculated to provide an indication of precision. The model based standard error is mean of the square of the bias, and the empirical standard error represents the spread of the estimates. This gives an indication of how much the point estimates vary across simulations based on the level of reporting and sample size. Although the method may give unbiased estimates with good coverage under repeated sampling, an imprecise method could lead to large differences from the true value when applied to a single dataset (that is, confidence intervals may cover the true value honestly but are wide).

We further explored the impact of bias and precision of the estimator by considering the deviations of the estimates from the true value termed absolute error. The absolute error is defined as the absolute difference between the estimated reporting and its true value, expressed as percentages. For instance, estimates of $43 \%$ and $62 \%$ for a true reporting of $50 \%$ would correspond to absolute errors of $7 \%$ and $12 \%$, respectively. During a disease outbreak, decisions are frequently made in the face of large uncertainties, and small absolute differences in the estimated level of reporting are unlikely to result in strategic changes. Therefore, as a perhaps more operationally relevant metric, we categorised results according to how far from the true value estimates were, using an arbitrary scale: very close ( $\leq 5 \%$ absolute error), close $(\leq 10 \%)$, approximate $(\leq 15 \%)$ or inaccurate $(\leq 20 \%)$. 


\section{Results}

\section{Bias}

There was very little bias across all the simulated scenarios (Table 3 and Figure 2). For outbreaks with over 100 cases all estimates of bias were 0 with decreasing Monte Carlo error from 0.04 to 0.01 as the size of the reported outbreak increased. For outbreaks reported as less than 100 cases the bias was -0.1 for reporting of 0.50 and 0.75 and 0 for 0.25 with Monte Carlo error of 0.07 . Table 3 presents the bias for each scenario and it can be seen that all of these estimates were within one standard error from zero, suggesting reasonable confidence that this is an overall unbiased estimator.

\section{Coverage}

The coverage varied across the simulated scenarios with all but reported outbreak size 10-99 with reporting at 0.25 displaying under-coverage (Figure 3 ). The coverage was poor with all coverage estimates more than one standard error away from 95\%, and most several standard errors away (Table 3). There was some suggestion of the counterintuitive pattern that coverage decreased as the reporting increased and that coverage decreased as the outbreak size increased. 


\section{Precision}

The model based standard error was below 0.07 for all estimates and below 0.04 for reported outbreaks of over 100 cases. Similar patterns are seen for the empirical standard error. Imprecise estimates were most marked when reported outbreaks were less than 100 cases and had 0.75 reporting. The precision increased (model based and empirical standard error decreased) as the reported outbreak size increased (Figure2, Table 3). Overall the precision appears reasonable when outbreaks are larger than 100.

\section{Absolute error}

Results showed that the estimates were rarely more than $15 \%$ away from the true reporting value in all simulation settings (Figure 4, Table 4). The absolute error was negligible in all larger reported outbreaks (500 cases and above), with the nearly all estimates very close (within $5 \%$ ) to the true reporting value. Performance decreased in smaller outbreaks, but most estimates remained close (within 10\%) to the true value. Results were worse in smaller outbreaks (10-99 reported cases), but even there about half of the estimates were very close (within $5 \%$ ) to the true value, and more than $80 \%$ of estimates were within $10 \%$ of the target. 


\section{Discussion}

We have presented a new estimator for the levels of reporting in an outbreak based on the proportion of cases with known infectors, which can be derived from case investigation data. Using simulated outbreaks to assess the performance of the method, we found that this approach generally had little bias, reasonable precision, but poor coverage. Across all simulations, estimated reporting was most often within $10 \%$ of the true value, suggesting the method will retain operational relevance under different settings.

Simulation results indicate a first limitation of the method lies in the analysis of smaller outbreaks. Overall, the approach performed better in larger outbreaks, with all metrics pointing to improved results in outbreaks of more than 100 reported cases. Our approach also assumes a uniform sampling of the transmission tree over the time period on which the analysis is carried. It would in theory be prone to under-estimating reporting when entire branches of the transmission tree remain unobserved. For instance, if an epidemic is spreading in a location where surveillance is totally absent, a substantial number of cases may remain unnoticed, and such under-reporting would not be accounted for in our estimates. As a consequence, our method is best applied to geographic areas over which surveillance and case investigation efforts do not vary drastically.

We also assumed that the reproduction number $(R)$ was independent from the reporting process, so that reported source cases cause the same average number of secondary cases as non-reported ones. This condition may not always be met, for instance if unreported individuals 
tend to cause more super-spreading events. In the context of Ebola, this may occur through community deaths, in which funeral exposure of a large number of relatives may give rise to a new cluster of cases from a single, unreported source case. Under such circumstances, we would expect our method to under-estimate reporting, although this should be further quantified by dedicated simulation studies.

Another limitation of our method relates to data availability and quality. Our approach relies on case investigation data, a time-consuming process usually requiring interviews of patients and/or their close relatives. There are several possible outcomes from such investigation: i) a single infector can be identified amongst reported cases ii) a single infector can be identified, but is not amongst reported cases iii) there are several likely infectors, possibly mixing reported and unreported cases iv) likely infector(s) could not be identified. Our approach requires case investigations to fall within the first two categories. In our simulations, we assumed for simplicity that all reported cases were successfully investigated, so that the reported outbreak size effectively corresponds to the number of data points available for the estimation. In practice, the actual sample size will be the number of case investigations which led to identifying a single source case (reported, or not). As our method performs better in larger datasets (e.g. more than 100 cases successfully investigated), the data requirement for estimating reporting from transmission chains will involve substantial field work.

Unfortunately, alternative approaches for estimating under-reporting are very demanding in terms of data, typically needing to combine information on dates of onset, location of the cases, full genome sequences of the pathogen for nearly all cases, good prior knowledge on key delays (e.g. incubation period, serial interval) $[13,16]$, and ideally contact tracing data [14]. 
These methodologies are also much more complex and computer-intensive, as they either involve the reconstruction of transmission trees $[13,14]$ or of outbreak clusters [16]. In contrast, the approach introduced here is fast and simple, and can be used in real time to estimate reporting based on data routinely collected as part of contact tracing activities and surveillance.

We evaluated the performance of the method using simulated EVD outbreaks in line with estimates of transmissibility and epidemiological delays of the Eastern DRC Ebola epidemic $[12,28]$, as this was the original context in which the method was developed. Further work should be devoted to investigating the method's performance for other diseases and different epidemic contexts. In particular, it would be interesting to study the potential impact of correlations between transmissibility and under-reporting, i.e. situations in which non-reported cases may exhibit increased infectiousness and cause super-spreading events.

\section{Conclusion}

In this paper, we provided a derivation of a straightforward and pragmatic estimator to real-time estimation of case reporting in outbreak settings, and tested this approach under a range of simulated conditions. The method exhibited little bias, reasonable precision, and while coverage was suboptimal under some settings (in large outbreaks with higher reporting), most estimates were within reasonable range $(10-15 \%)$ of the true value. This suggests the method will be useful for informing the response to outbreaks in which person-to-person transmission is the main driver of transmission, and where enough (ideally $>100$ ) chains of transmissions can be retraced through epidemiological investigation. 


\section{Acknowledgements}

TJ, CIJ and WJE receive funding from the Global Challenges Research Fund (GCRF) project 'RECAP' managed through RCUK and ESRC (ES/P010873/1). TJ and OPW receive funding from the UK Public Health Rapid Support Team funded by the United Kingdom Department of Health and Social Care. TJ receives funding from the National Institute for Health Research Health Protection Research Unit for Modelling Methodology. The authors alone are responsible for the views expressed in this article and they do not necessarily represent the views, decisions or policies of the institutions with which they are affiliated. TPM was funded by the UK MRC (grants MC_UU_12023/21 and MC_UU_12023/29). JAT was jointly funded by the UK Medical Research Council (MRC) and the UK Department for International Development (DFID) under the MRC/DFID Concordat agreement and is also part of the EDCTP2 programme supported by the European Union (MR/R010161/1)

The UK Public Health Rapid Support Team is funded by the United Kingdom Department of Health and Social Care. The views expressed in this publication are those of the authors and not necessarily those of the National Health System, the National Institute for Health Research or the Department of Health and Social Care.

We are thankful to the R Epidemics Consortium (RECON) for developing free software tools used in the simulations studies. 


\section{Author Contribution}

(In alphabetic order)

Conceptualization: AG, CIJ, TJ

Methodology: CIJ, FF, SF, TJ, TPM, JAT

Formal Analysis, software and visualization: AG, CIJ, TJ

Writing - Original Draft Preparation: AG, CIJ, TJ

Writing - Review \& Editing: WJE, FF, SF, TJ, TPM, JAT, OW

All authors read and approved the final version of the manuscript. 


\section{Figures}

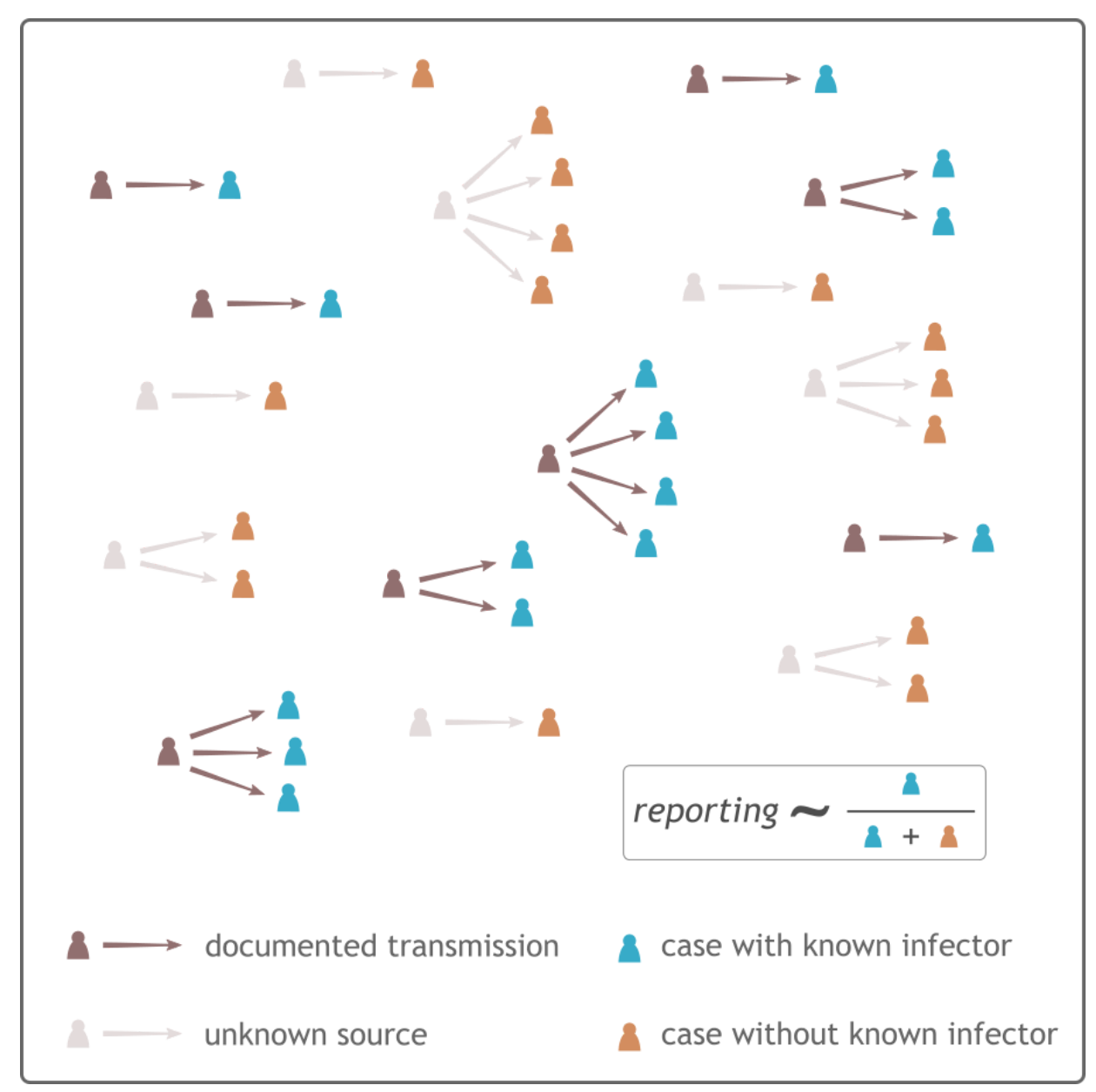

Figure 1: rationale of the method for estimating reporting. This diagram illustrates transmission events inferred by case investigation of reported secondary cases, with arrows pointing from infectors to infectees. Darker shades are used to indicate documented transmission events, while lighter shades show unknown infectors. Numbers of secondary cases with (blue) or without (orange) known infectors are used to estimate the reporting probability. This example uses an approximate reporting of $50 \%$. 


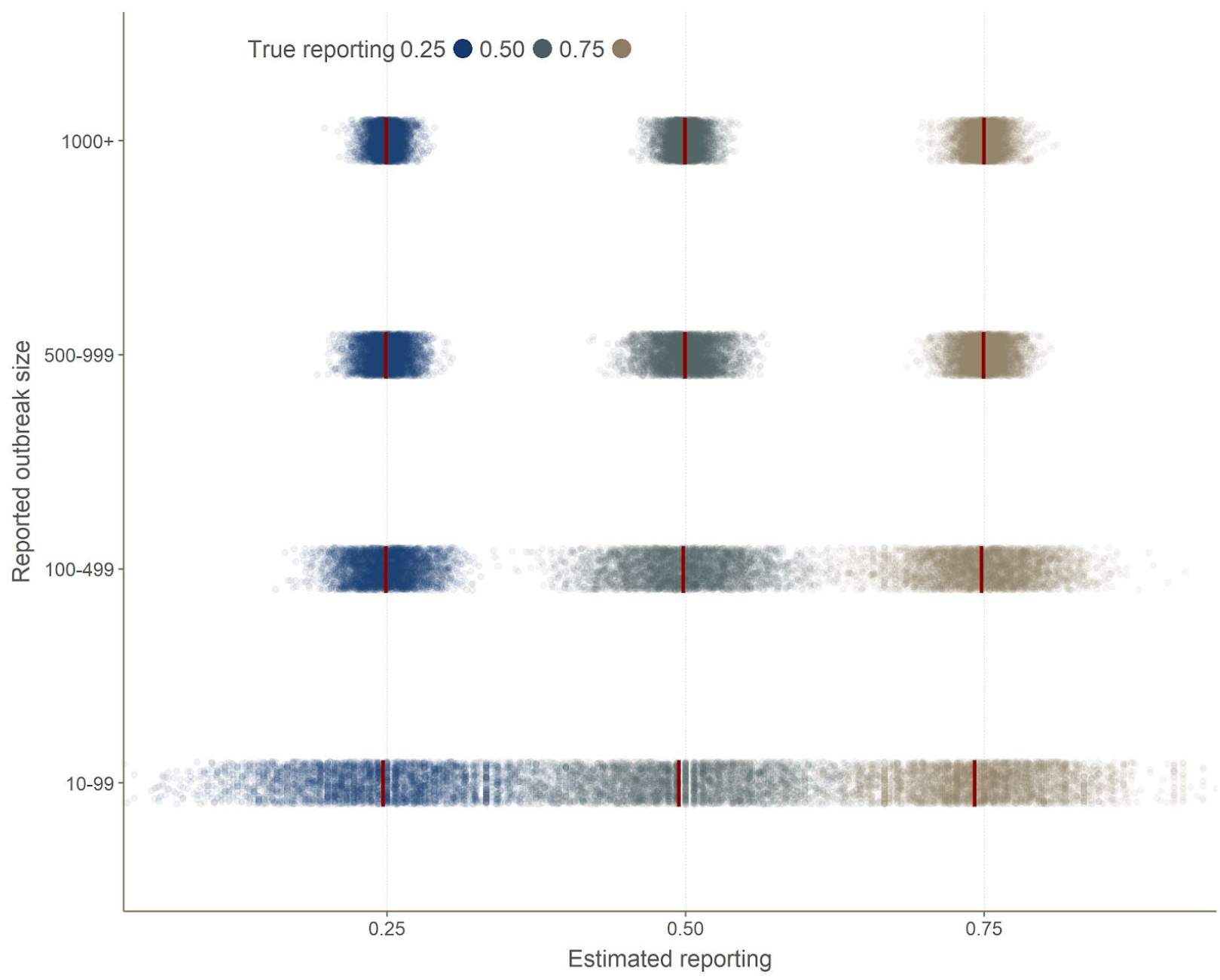

Figure 2: comparison of estimated versus actual reporting. This graph shows the results of reporting estimated by the method for 4000 simulated outbreaks, broken down by outbreak size category (y-axis). Each dot corresponds to an independent simulation. The vertical red bars indicate the average within each category. True reporting used in the simulations is indicated by colors. 


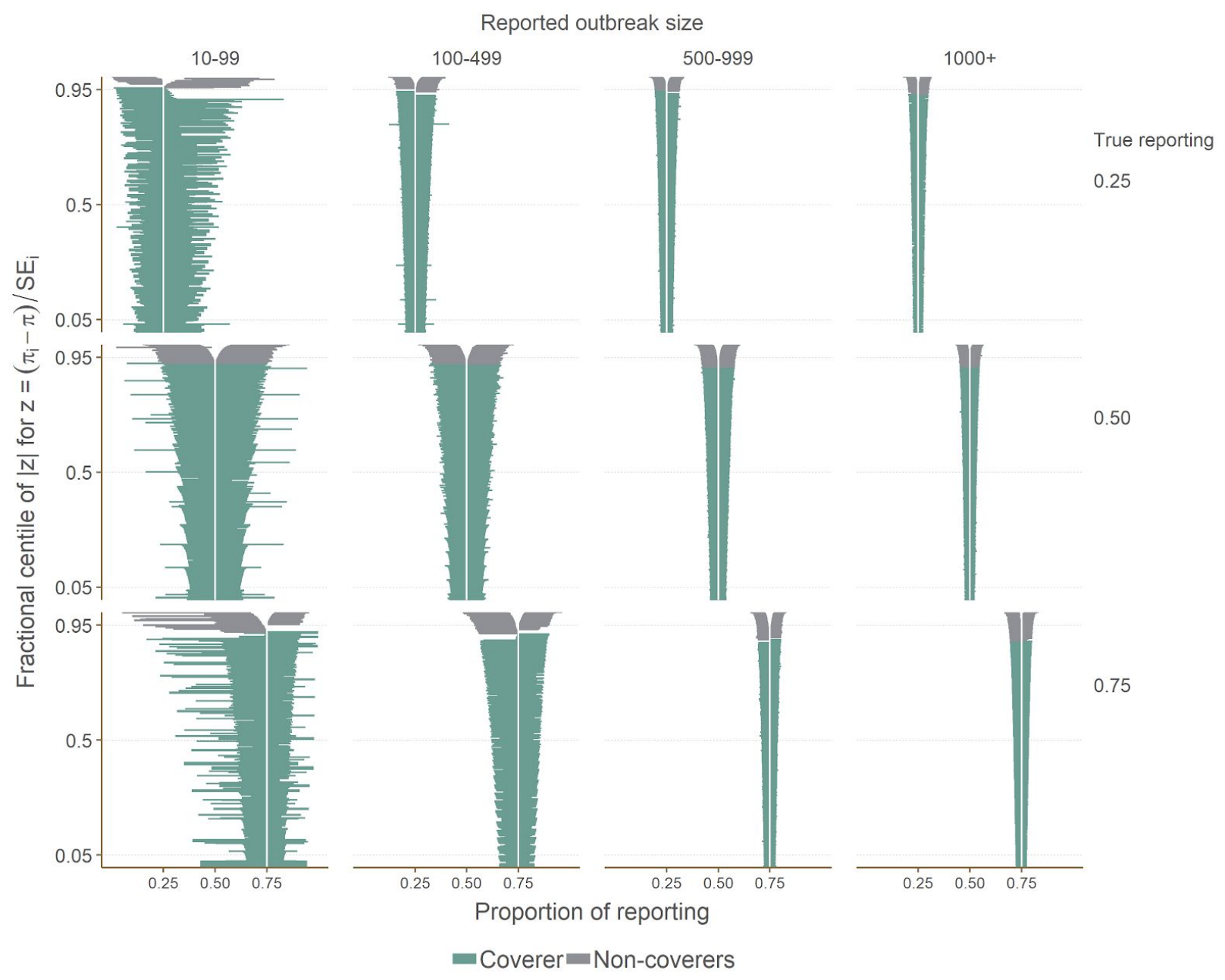

Figure 3: Zip plot of showing coverage results. This graph shows the $95 \%$ confidence intervals estimated by the method, broken down by reported outbreak size category and true reporting value. The vertical axis represent the fractional centile of $|Z|$ where $Z=\frac{\left(\pi_{i}-\pi\right)}{S E_{i}}$ and $\pi$ is reporting. The confidence intervals are ranked by their level of coverage and thus the vertical axis can be used to determine the proportion of confidence intervals that contain the true value where 0.95 would represent a coverage of $95 \%$. 


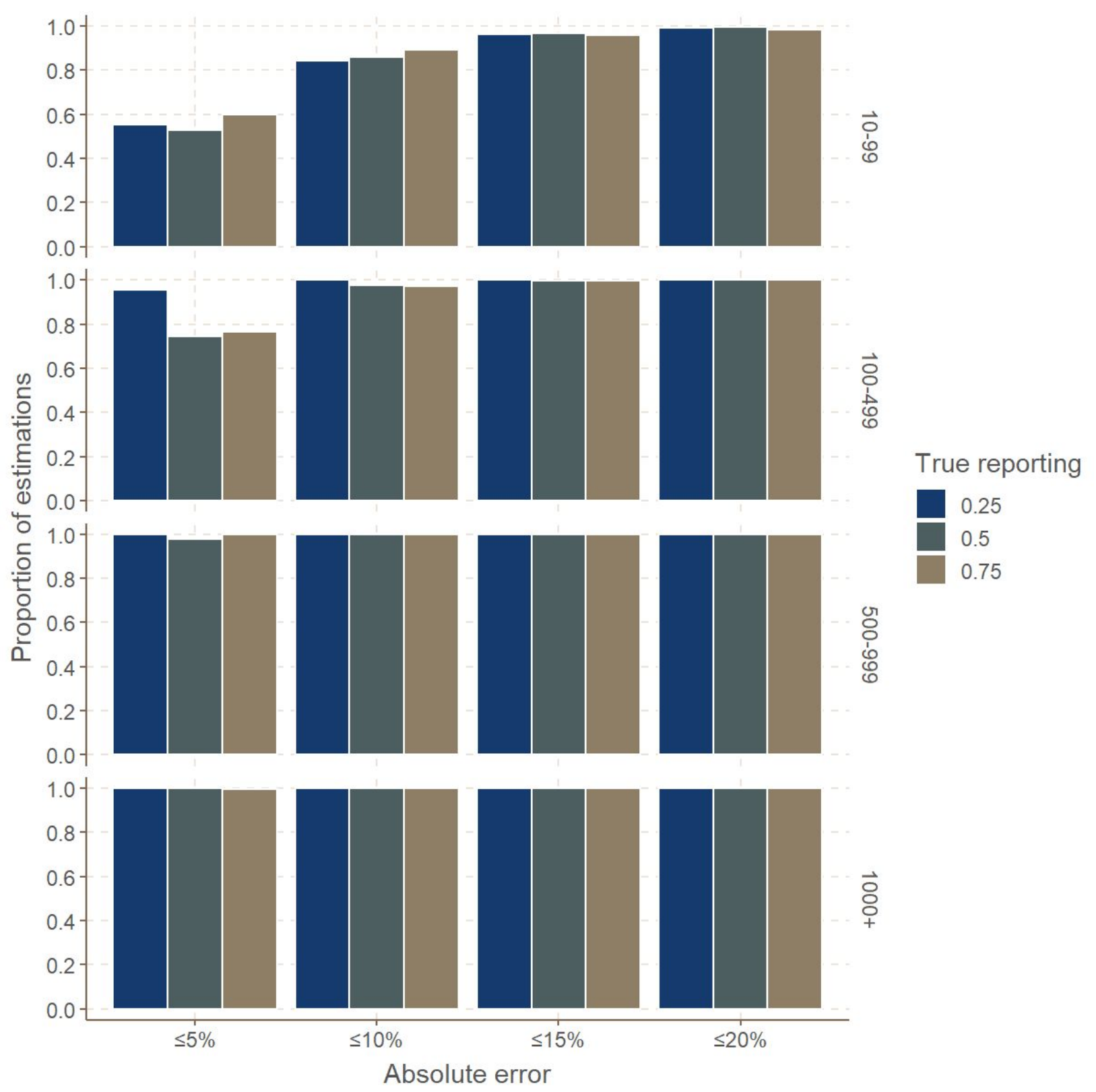

Figure 4: Absolute error in reporting estimation. This graph shows, for different simulation settings, the proportion of results within a given margin of absolute error, expressed as the absolute difference between the true and the estimated reporting (in \%). Rows correspond to different outbreak size categories (outbreak size as reported). True reporting is indicated in color. 


\section{Tables}

Table 1: Parameters used for simulating outbreaks. This table details input parameters used

for simulating outbreaks using the R package simulacr. Fixed values were used for all

simulations, and reflect the natural history of the 2018-2020 Eastern DRC Ebola outbreak.

Variable values changed across simulations.

\section{Fixed values}

\begin{tabular}{|c|c|}
\hline Maximum duration of the outbreak & 365 days \\
\hline Incubation time distribution & $\begin{array}{l}\text { Discretised gamma distribution } \\
\text { mean of } 9.7 \text { days, } \mathrm{sd}=5.5 \text { days. }\end{array}$ \\
\hline Infectious period distribution & $\begin{array}{l}\text { Discretised gamma distribution } \\
\text { mean }=5 \text { days, } \mathrm{sd}=4.7 \text { days. }\end{array}$ \\
\hline Reproduction number distribution & $\begin{array}{l}\text { Gamma distribution: } \\
\text { rate of } 1.2 \text { shape of } 2 .\end{array}$ \\
\hline
\end{tabular}

\section{Variable values}

$\begin{array}{ll}\text { Population size }^{*} & 200,500,1000,2000,5000,7500,10000,15000,20000 \\ & \\ \text { Outbreak size* } & 10-99,100-499,500-999,1000+ \\ \text { Proportion of cases not reported } & 0.25,0.50,0.75\end{array}$

${ }^{*}$ Population size is controlled in each simulation, the outbreak sizes are determined after the outbreaks have been simulated and the proportion of cases not reported have been removed. 
Table 2: Metrics used to measure performance in the simulation study.

\begin{tabular}{ll}
\hline Performance measure & Definition \\
\hline Bias & $\delta=E[\widehat{\theta}]-\theta$ where $\theta$ is the true value and $\widehat{\theta}$ is the estimate of value \\
Coverage & If we define a confidence interval $\left(\widehat{\theta}_{\text {low }}, \widehat{\theta}_{\text {upp }}\right)$ as the \\
& $P\left(\widehat{\theta}_{\text {low }} \leq \theta \leq \widehat{\theta}_{\text {upp }}\right)=\psi$ where $\psi \in[0,1]$ then a $95 \% \mathrm{Cl}$ is when \\
& $P\left(\widehat{\theta}_{\text {low }} \leq \theta \leq \widehat{\theta}_{\text {upp }}\right)=0.95$. It follows that coverage is the \\
& $P\left(\widehat{\theta}_{\text {low }} \leq \theta \leq \widehat{\theta}_{\text {upp }}\right)$. \\
Precision \\
$\begin{array}{l}\text { Model based } \\
\text { standard error }\end{array}$ \\
$\begin{array}{l}\text { Empirical based } \\
\text { standard error }\end{array}$ \\
$\qquad\left[\left(\widehat{V}^{\operatorname{\theta ar}(\theta)}-\theta\right)^{2}\right]$
\end{tabular}

Absolute error $\quad\left|\hat{\theta}_{i}-\theta\right|$


Table 3: Performance measures from 4000 simulation by reported outbreak size and true reporting level. Estimate (Monte-carlo standard error)

\begin{tabular}{|c|c|c|c|c|c|}
\hline \multirow[b]{2}{*}{ Performance measures (MCSE) } & \multirow[b]{2}{*}{ Proportion reported } & \multicolumn{4}{|c|}{ Reported outbreak size } \\
\hline & & $10-99$ & $100-499$ & $500-999$ & 1000 or more \\
\hline \multirow[t]{3}{*}{ Bias } & 0.25 & $0(0.07)$ & $0(0.03)$ & $0(0.02)$ & $0(0.01)$ \\
\hline & 0.5 & $-0.01(0.07)$ & $0(0.04)$ & $0(0.02)$ & $0(0.01)$ \\
\hline & 0.75 & $-0.01(0.07)$ & $0(0.04)$ & $0(0.02)$ & $0(0.01)$ \\
\hline \multirow[t]{3}{*}{ Coverage } & 0.25 & $95.7 \%(0.3)$ & $94.1 \%(0.4)$ & $94.4 \%(0.4)$ & $93 \%(0.4)$ \\
\hline & 0.5 & $92.6 \%(0.4)$ & $92.4 \%(0.4)$ & $91.3 \%(0.4)$ & $91.2 \%(0.4)$ \\
\hline & 0.75 & $92.3 \%(0.4)$ & $91.5 \%(0.4)$ & $89.2 \%(0.5)$ & $88.6 \%(0.5)$ \\
\hline \multirow[t]{3}{*}{ Model standard error } & 0.25 & $0.065(0)$ & $0.024(0)$ & $0.015(0)$ & $0.01(0)$ \\
\hline & 0.5 & $0.061(0)$ & $0.038(0)$ & $0.019(0)$ & $0.011(0)$ \\
\hline & 0.75 & $0.059(0.001)$ & $0.036(0)$ & $0.014(0)$ & $0.011(0)$ \\
\hline \multirow[t]{3}{*}{ Empirical standard error } & 0.25 & $0.071(0.001)$ & $0.025(0)$ & $0.016(0)$ & $0.01(0)$ \\
\hline & 0.5 & $0.07(0.001)$ & $0.044(0)$ & $0.022(0)$ & $0.012(0)$ \\
\hline & 0.75 & $0.068(0.001)$ & $0.043(0)$ & $0.017(0)$ & $0.013(0)$ \\
\hline
\end{tabular}


Table 4: Comparison of absolute error from 4000 simulations between true reporting

levels and estimate of reporting by reported outbreak size and true reporting level

\begin{tabular}{|c|c|c|c|c|c|}
\hline \multirow[b]{2}{*}{ Proportion reported } & \multirow[b]{2}{*}{ Reported outbreak size } & \multicolumn{4}{|c|}{ Absolute error from true value } \\
\hline & & $\leq 5 \%$ & $\leq 10 \%$ & $\leq 15 \%$ & $\leq 20 \%$ \\
\hline \multirow[t]{4}{*}{0.25} & $10-99$ & $2213(55.3 \%)$ & $3376(84.4 \%)$ & 3849 (96.2\%) & $3973(99.3 \%)$ \\
\hline & $100-499$ & 3817 (95.4\%) & 4000 & 4000 & 4000 \\
\hline & $500-999$ & 3995 (99.9\%) & 4000 & 4000 & 4000 \\
\hline & $1000+$ & 3999 (100\%) & 4000 & 4000 & 4000 \\
\hline \multirow[t]{4}{*}{0.5} & $10-99$ & $2110(52.8 \%)$ & $3430(85.8 \%)$ & 3860 (96.5\%) & 3978 (99.4\%) \\
\hline & $100-499$ & 2981 (74.5\%) & $3899(97.5 \%)$ & $3998(100 \%)$ & 4000 \\
\hline & $500-999$ & 3905 (97.6\%) & 4000 & 4000 & 4000 \\
\hline & $1000+$ & 4000 & 4000 & 4000 & 4000 \\
\hline \multirow[t]{4}{*}{0.75} & $10-99$ & $2400(60 \%)$ & 3575 (89.4\%) & 3835 (95.9\%) & $3942(98.6 \%)$ \\
\hline & $100-499$ & 3067 (76.7\%) & $3890(97.2 \%)$ & 3991 (99.8\%) & 4000 \\
\hline & $500-999$ & 3988 (99.7\%) & 4000 & 4000 & 4000 \\
\hline & $1000+$ & 3992 (99.8\%) & 4000 & 4000 & 4000 \\
\hline
\end{tabular}




\section{References}

1. Cori A, Donnelly CA, Dorigatti I, Ferguson NM, Fraser C, Garske T, et al. Key data for outbreak evaluation: building on the Ebola experience. Philos Trans R Soc Lond B Biol Sci. 2017;372. doi:10.1098/rstb.2016.0371

2. Polonsky JA, Baidjoe A, Kamvar ZN, Cori A, Durski K, John Edmunds W, et al. Outbreak analytics: a developing data science for informing the response to emerging pathogens. Philosophical Transactions of the Royal Society B: Biological Sciences. 2019. p. 20180276. doi:10.1098/rstb.2018.0276

3. Dalziel BD, Lau MSY, Tiffany A, McClelland A, Zelner J, Bliss JR, et al. Unreported cases in the 2014-2016 Ebola epidemic: Spatiotemporal variation, and implications for estimating transmission. PLoS Negl Trop Dis. 2018;12: e0006161.

4. Meltzer MI, Atkins CY, Santibanez S, Knust B, Petersen BW, Ervin ED, et al. Estimating the future number of cases in the Ebola epidemic--Liberia and Sierra Leone, 2014-2015.

MMWR Suppl. 2014;63: 1-14.

5. World Health Organisation (WHO). Guinea: The Ebola Virus Shows its Tenacity. In: WHO [Internet]. 2015 [cited 20 Jan 2020]. Available:

https://www.who.int/csr/disease/ebola/one-year-report/guinea/en/

6. Mercer GN, Glass K, Becker NG. Effective reproduction numbers are commonly overestimated early in a disease outbreak. Stat Med. 2011;30: 984-994.

7. Bloch A. A serological survey of Lassa fever in Liberia. Bull World Health Organ. 1978;56: 811-813.

8. Gérardin P, Guernier V, Perrau J, Fianu A, Le Roux K, Grivard P, et al. Estimating Chikungunya prevalence in La Réunion Island outbreak by serosurveys: Two methods for two critical times of the epidemic. BMC Infect Dis. 2008;8: 99.

9. Farnon EC, Laven JJ, Hayes EB, Griffith KS, Godsey MS, Panella AJ, et al. Household-Based Sero-Epidemiologic Survey after a Yellow Fever Epidemic, Sudan, 2005. The American Journal of Tropical Medicine and Hygiene. 2010. pp. 1146-1152. doi:10.4269/ajtmh.2010.09-0105

10. Aylward B, Barboza P, Bawo L, Bertherat E, Bilivogui $P$, Blake I, et al. Ebola virus disease in West Africa--the first 9 months of the epidemic and forward projections. $\mathrm{N}$ Engl $\mathrm{J}$ Med. 2014;371: 1481-1495.

11. Ilunga Kalenga $O$, Moeti M, Sparrow A, Nguyen V-K, Lucey D, Ghebreyesus TA. The Ongoing Ebola Epidemic in the Democratic Republic of Congo, 2018-2019. N Engl J Med. 2019;381: 373-383. 
12. Jombart T, Jarvis Cl, Mesfin S, Tabal N, Mossoko M, Mpia LM, et al. The cost of insecurity: from flare-up to control of a major Ebola virus disease hotspot during the outbreak in the Democratic Republic of the Congo, 2019. Eurosurveillance. 2020. doi:10.2807/1560-7917.es.2020.25.2.1900735

13. Jombart T, Cori A, Didelot X, Cauchemez S, Fraser C, Ferguson N. Bayesian reconstruction of disease outbreaks by combining epidemiologic and genomic data. PLoS Comput Biol. 2014;10: e1003457.

14. Campbell $F$, Cori $A$, Ferguson $N$, Jombart $T$. Bayesian inference of transmission chains using timing of symptoms, pathogen genomes and contact data. PLoS Comput Biol. 2019;15: e1006930.

15. Campbell F, Didelot X, Fitzjohn R, Ferguson N, Cori A, Jombart T. outbreaker2: a modular platform for outbreak reconstruction. BMC Bioinformatics. 2018;19: 363.

16. Cori A, Nouvellet P, Garske T, Bourhy H, Nakouné E, Jombart T. A graph-based evidence synthesis approach to detecting outbreak clusters: An application to dog rabies. PLOS Computational Biology. 2018. p. e1006554. doi:10.1371/journal.pcbi.1006554

17. Scarpino SV, lamarino A, Wells C, Yamin D, Ndeffo-Mbah M, Wenzel NS, et al. Epidemiological and viral genomic sequence analysis of the 2014 ebola outbreak reveals clustered transmission. Clin Infect Dis. 2015;60: 1079-1082.

18. Gamado KM, Streftaris G, Zachary S. Modelling under-reporting in epidemics. J Math Biol. 2014;69: 737-765.

19. Gamado K, Streftaris G, Zachary S. Estimation of under-reporting in epidemics using approximations. J Math Biol. 2017;74: 1683-1707.

20. Barry A, Ahuka-Mundeke S, Ali Ahmed Y, Allarangar Y, Anoko J, Archer BN, et al. Outbreak of Ebola virus disease in the Democratic Republic of the Congo, April-May, 2018: an epidemiological study. Lancet. 2018;392: 213-221.

21. Bernard Stoecklin S, Rolland P, Silue $Y$, Mailles A, Campese $C$, Simondon A, et al. First cases of coronavirus disease 2019 (COVID-19) in France: surveillance, investigations and control measures, January 2020. Euro Surveill. 2020;25.

doi:10.2807/1560-7917.ES.2020.25.6.2000094

22. Donnelly CA, Ghani AC, Leung GM, Hedley AJ, Fraser C, Riley S, et al. Epidemiological determinants of spread of causal agent of severe acute respiratory syndrome in Hong Kong. Lancet. 2003;361: 1761-1766.

23. International Ebola Response Team, Agua-Agum J, Ariyarajah A, Aylward B, Bawo L, Bilivogui P, et al. Exposure Patterns Driving Ebola Transmission in West Africa: A Retrospective Observational Study. PLoS Med. 2016;13: e1002170.

24. Morris TP, White IR, Crowther MJ. Using simulation studies to evaluate statistical methods. Stat Med. 2019;38: 2074-2102. 
25. Burton A, Altman DG, Royston P, Holder RL. The design of simulation studies in medical statistics. Stat Med. 2006;25: 4279-4292.

26. simulacr. Github; Available: https://github.com/reconhub/simulacr

27. Campbell F, Strang C, Ferguson N, Cori A, Jombart T. When are pathogen genome sequences informative of transmission events? PLoS Pathog. 2018;14: e1006885.

28. Kalenga OI, Moeti M, Sparrow A, Nguyen V-K, Lucey D, Ghebreyesus TA. The Ongoing Ebola Epidemic in the Democratic Republic of Congo, 2018-2019. New England Journal of Medicine. 2019. pp. 373-383. doi:10.1056/nejmsr1904253

29. Koehler E, Brown E, Haneuse SJ-PA. On the Assessment of Monte Carlo Error in Simulation-Based Statistical Analyses. Am Stat. 2009;63: 155-162. 
bioRxiv preprint doi: https://doi.org/10.1101/2021.02.17.431606; this version posted February 17, 2021. The copyright holder for this preprint (which was not certified by peer review) is the author/funder, who has granted bioRxiv a license to display the preprint in perpetuity. It is made available under aCC-BY 4.0 International license. 